\title{
Aberration Corrected Lorentz Microscopy to Investigate Magnetic Domain Walls in Co-Pt Nano-Chessboards
}

\author{
Isha Kashyap ${ }^{1}$, Jerrold A. Floro ${ }^{2}$, Yongmei M. Jin ${ }^{3}$ and Marc De Graef ${ }^{1}$ \\ 1. Dept. of Materials Science and Engineering, Carnegie Mellon University, Pittsburgh, USA. \\ 2. Dept. of Materials Science and Engineering, University of Virginia, Charlottesville, USA. \\ 3. Dept. of Materials Science and Engineering, Michigan Technological University, Houghton, USA.
}

Lorentz Transmission Electron Microscopy (LTEM) is a technique used to image and characterize magnetic domains and magnetic domain walls. In LTEM mode, since the sample sits inside the objective lens, the lens is turned off in order to preserve the magnetic structure of the sample. A separate Lorentz lens is used to image the sample, albeit with in a great reduction in attainable magnification. The conventional LTEM offers a spatial resolution of $\sim 10-15 \mathrm{~nm}$ and requires a very high defocus value (of the order of several microns) to make magnetic domain walls visible, which further degrades the resolution of the final images; this makes it challenging to study nano-scale magnetic structures. However, recent breakthroughs in aberration corrected microscopy have enabled the study of complex and fine scale magnetic domains in the Lorentz TEM mode. In this study, we use an FEI Titan 80-300 microscope with a dedicated Lorentz pole piece that offers $\mathrm{C}_{\mathrm{S}}$-corrected field free imaging $(<2$ Oe) with $<1 \mathrm{~nm}$ resolution. The Fresnel or defocus mode of Lorentz imaging is employed to investigate domain walls. The Fresnel mode LTEM only provides qualitative information about the position of domain walls. Quantitative information about the magnetic induction can be extracted from a Fresnel throughfocus series of images (in-focus, over-focus, and under-focus) by reconstructing the phase of the electron waves using the transport-of-intensity equation. Aberration correction also reduces the defocus value required to image the domain walls and makes Fresnel images useful for quantitative analysis. A detailed description of Lorentz Fresnel mode and phase reconstruction can be found in [1].

Co-Pt ordered alloys of near-eutectoid compositions $\left(\sim \mathrm{Co}_{40} \mathrm{Pt}_{60}\right)$ undergo eutectoid decomposition to produce a nano-chessboard structure, which is composed of two interleaved phases - the hard magnetic $\mathrm{L}_{0}$ phase $(\mathrm{CoPt})$ and the soft magnetic $\mathrm{L}_{2}$ phase $\left(\mathrm{CoPt}_{3}\right)$. This material is expected to have potential for high density magnetic storage due to the exchange coupling between the hard and the soft magnetic phases. We use $\mathrm{C}_{\mathrm{S}}$-corrected Lorentz $\mathrm{TEM}$ in combination with micromagnetic simulations to characterize magnetic domain walls in $\mathrm{Co}_{40.2} \mathrm{Pt}_{59.8}$ nano-chessboards. The output magnetization from the micromagnetic simulations is used to compute the magnetic phase shift of the electron wave using the Mansuripur algorithm [2,3]. Fresnel images and color integrated induction maps are simulated using the computed phase shift. A series of color maps is obtained for different beam tilts and magnetization states. Due to limitations in operating the LTEM in diffraction mode, the experimental Fresnel images are acquired at unknown sample orientations. Since a clockwise sample tilt inside the TEM is equivalent to an anti-clockwise tilt of the electron beam, the magnetic contrast in the experimentally obtained color map can be analyzed by comparing it with simulated color maps generated at different beam tilts.

Fig. 1(a) shows an experimental nano-chessboard TEM dark field image. The black tiles are the $\mathrm{L} 1_{0}$ phase and white tiles are the $\mathrm{L}_{2}$ phase. The easy axes of $\mathrm{L} 1_{0}$ tiles are labeled in the figure. Fig. 1(b) shows a Fresnel under-focus image. The zigzag or ' $S$ ' shaped magnetic domain walls are visible as white bright contrast in the under-focus image. The phase map and the color map of marked rectangular area are shown in Fig. 1(c) and Fig. 1(d), respectively. The color map represents the direction of 
integrated magnetic induction inside the sample. The overlaid magnetization vectors (white arrows) in Fig. 1(d) show the direction of magnetic induction in each of the $\mathrm{L} 1_{0}$ tiles. Fig. 2 shows the simulation results. The white magnetization vectors in Fig. 2(c) show a vortex-type pattern and the black magnetization vectors show an anti-vortex type pattern. The vortex type repetitive pattern can also be observed in the experimental color map. The type of magnetic domain arrangement is dependent on the relative orientation of the easy axis of magnetization with respect to the sample normal. Only the inplane component of magnetization gives rise to magnetic contrast. As the sample is tilted away from the zone axis [001], the in-plane magnetic component corresponding to each $\mathrm{L} 1_{0}$ tile changes and a nonsymmetrical distribution of colors is seen in the color map. This asymmetry in the magnetic contrast can be seen in the simulated color map obtained at $15^{\circ}$ beam tilt (Fig. 2(d)). The blue and yellow color contrast becomes more pronounced as the beam is tilted towards [010]. This is also evident in the experimental image. Future work involves extensive simulations combined with in-situ Lorentz imaging in order to understand exchange coupling in Co-Pt nano-chessboards.

\section{References:}

[1] M. De Graef, Introduction to conventional transmission electron microscopy (2003).

[2] Eric P. Vetter, Liwei Geng, Priya Ghatwai et al., APL Materials 4 (2016) 096103.

[3] M. Mansuripur, Journal of Applied Physics 69 No. 4 (1991) p.2455.

[4] Eric P. Vetter, Priya Ghatwai, William A. Soffa et al., IEEE Magnetic Letters 6 (2015) 6600104.

[5] Isha Kashyap and M. De Graef acknowledge the support by grant MCF-677785, Materials

Characterization Facility at Carnegie Mellon University. JAF acknowledges support of the National Science Foundation under grant DMR-1105336.
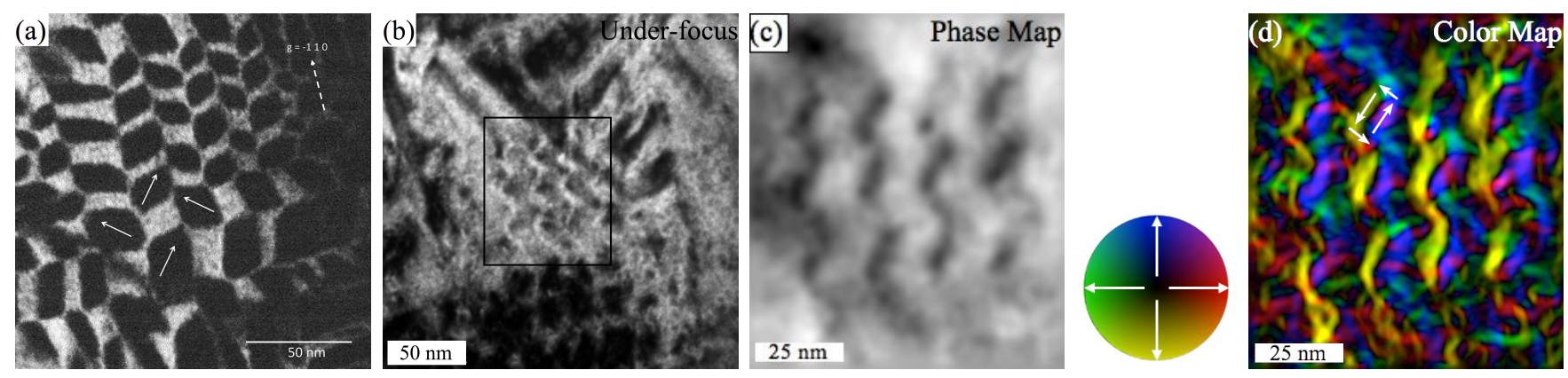

Figure 1. (a) Experimental nano-chessboard (-110) dark field image on [001] zone axis [4]; (b) Fresnel under-focus image $(\Delta \mathrm{f}=1 \mu \mathrm{m})$ showing the S-shaped domain walls; (c) Phase reconstructed map; (d) Color induction map showing the direction of magnetic induction inside each of the black tiles.
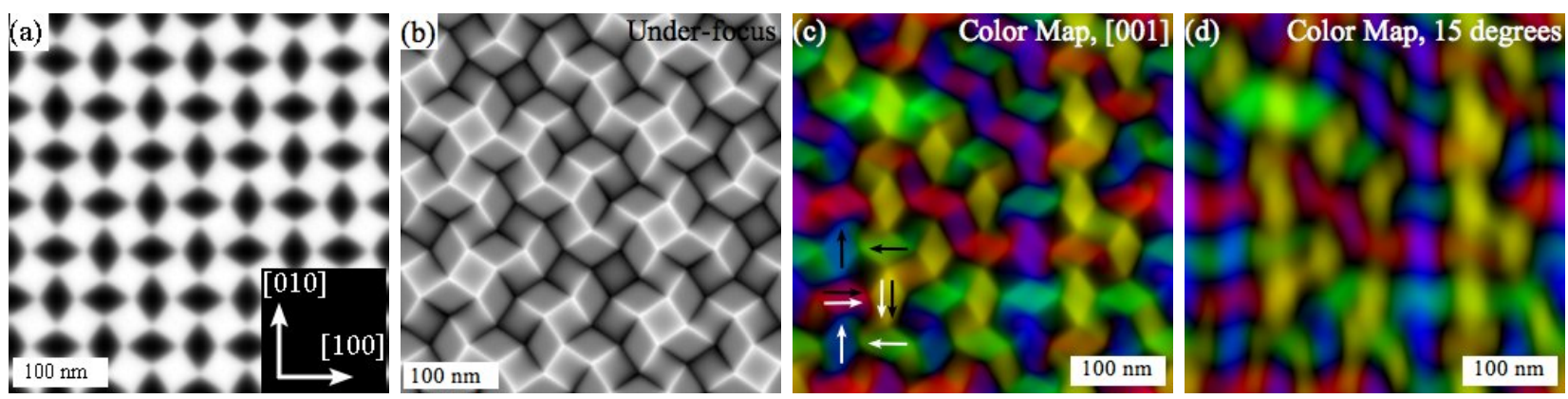

Figure 2. (a) Simulated 2-D nano-chessboard viewed along [001] direction; (b) Simulated Fresnel under-focus image showing zigzag domain wall contrast; (c) Color map at $0^{\circ}$ beam tilt, [001] zone axis; (d) Color map at $15^{\circ}$ beam tilt. 